\title{
CONSTRUCTION OF A RIGID ARONSZAJN TREE ${ }^{1}$
}

\author{
URI AVRAHAM
}

AbStract. We construct in ZFC an Aronszajn tree with no automorphism.

Using the nonisomorphic Aronszajn trees constructed by Gaifman and Specker [1] we build in ZFC a normal rigid Aronszajn tree thus answering a question of T. J. Jech [2].

For a tree $T$ and $a \in T, T a$ denotes the part of $T$ above $a$, i.e., $T a=\{x \in$ $T \mid x \geqslant a\} . T_{\alpha}, \alpha<\omega_{1}$, is the $\alpha$ th level of $T$. We say that $a, b \in T$ meet at the level $\alpha$ if there is an $e \in T_{\alpha}, e<a, e<b$, but no such $e$ exists in $T_{\alpha+1}$.

By [1] we have for every uncountable $X \subseteq \omega_{1}$ a normal Aronszajn tree $T(X)$ such that:

(1) for every $a \in T(X)$ there is an uncountable subset of $T(X)_{a}$ every two elements of which meet at level in $X$, and

(2) there does not exist an uncountable subset of $T(X)$ every two elements of which meet at level in $\omega_{1}-X$.

(Briefly, if we start from an Aronszajn tree $T \subseteq \omega^{\omega_{1}}$ then $T(X) \subseteq \omega^{\omega_{1}}$ is the set of all functions $f$ such that the collapse of $f \mid X$ is in $T$ and $f \mid\left(\omega_{1}-X\right)$ is different from zero only finitely many times.)

We will construct a normal Aronszajn tree $R$ such that there is no order preserving function $f: R \rightarrow R$ other than the identity. Let $X, X_{\alpha, n}, \alpha<\omega_{1}$, $n<\omega$, be pairwise disjoint uncountable subsets of $\omega_{1}$, construct the trees $T\left(X_{\alpha, n}\right)$ such that their domain will be pairwise disjoint. We construct a sequence $R^{n}, n<\omega$, of Aronszajn trees such that $R^{n} \subseteq R^{n+1}$ by induction on $n$. A point in $R^{n+1}-R^{n}$ is said to be of rank $n+1$.

$R^{0}$ is $T(X)$. Suppose $R^{n}$ is defined, let $\left\{a_{\gamma}^{n} \mid \gamma<\omega_{1}\right\}$ be its elements, for every $a_{\gamma}^{n}, a_{\gamma}^{n} \in R_{\alpha}^{n}$ for some $\alpha<\omega_{1}$, take any member $b_{\gamma}^{n}$ of $T\left(X_{\gamma, n}\right)_{\alpha+1}$ and plant $T\left(X_{\gamma, n}\right)_{b_{\gamma}^{n}}$ above $a_{\gamma}^{n}$. The result is $R^{n+1}$. Again, $R^{n+1}$ is obtained by partial ordering $R^{n} \cup \cup_{\gamma<\omega_{1}} T\left(X_{\gamma, n}\right)_{b_{\gamma}^{n}}$ as follows: for elements of $R^{n}$ or $T\left(X_{\gamma, n}\right)$ the order is as in $R^{n}$ or $T\left(X_{\gamma, n}\right)$, respectively, $b_{\gamma}^{n}$ is above $a_{\gamma}^{n}$ and the partial order is transitive. For $x \in T\left(X_{\gamma, n}\right)$ we define $r(x)=b_{\gamma}^{n}$ and $r^{*}(x)=$ $a_{\gamma}^{n}$, for $x \in R^{0}, r(x)$ is the root of $R^{0}$.

$R=\cup_{n<\omega} R^{n}$ is a normal Aronszajn tree. Note that for $m<n$ an element of rank $n$ cannot be below an element of rank $m$. We prove that $R$ is

Received by the editors February 8, 1978.

AMS (MOS) subject classifications (1970). Primary 04A20.

Key words and phrases. Aronszajn tree, rigid tree.

${ }^{1}$ ADDED IN PROOF. This result was already known to J. Baumgartner. See Notices Amer. Math. Soc. 22 (1975), p. A-219. 
rigid. Let $f: R \rightarrow R$ be order preserving and for some distinct $x, y \in R_{\alpha}$, $f(x)=y$.

For some $n<\omega, x, y \in R^{n}, x=a_{\gamma}^{n}$ for some $\gamma<\omega_{1}$ and $T\left(X_{\gamma, n}\right)_{b_{\gamma}^{n}}$ was grafted above $a_{\gamma}^{n}$, it follows from (1) that we can find $\aleph_{1}$ elements in $R_{f(x)}$ every two of which meet at level in $X_{\gamma, n}$. Let $k<\omega$ be the least integer such that we can find an uncountable $A \subseteq R_{y}^{k}$ every two elements of which meet at level in $X_{\gamma, n}$. Look at $B=\left\{r^{*}(x) \mid x \in A\right\}$ for such an $A$. If it is uncountable then we have $\aleph_{1}$ elements in $R_{y}^{k-1}$ contradicting the definition of $k$. Hence we can find $b$ such that $b=r(x)$ for uncountably many $x$ in $A$. $b$ is compatible with $y$, hence distinct from $x$ so that $b=b_{\eta}^{l}$ for some $(\eta, l)$ distinct from $(\gamma, n)$ it follows that we have found $\kappa_{1}$ elements in $T\left(X_{\eta, l}\right)$ every two of which meet at level outside $X_{\eta, l}$, and this is a contradiction.

\section{REFERENCES}

1. H. Gaifman and E. P. Specker, Isomorphism types of trees, Proc. Amer. Math. Soc. 15 (1964), $1-7$.

2. T. J. Jech, Automorphism of $\omega_{1}$-trees, Trans. Amer. Math. Soc. 173 (1972), 57-70.

Department of Mathematics, The Hebrew University of Jerusalem, Jerusalem, Israel

Current address. Department of Mathematics, Dartmouth College, Hanover, New Hampshire 03755 